
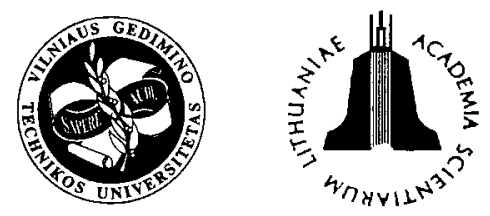

ISSN $1392-3730$

JOURNAL OF CIVIL ENGINEERING AND MANAGEMENT

http:/www.vtu.lt/english/edition

2002, Vol VIII, No 2, 83-87

\title{
RELIABILITY OF IMPERFECT STRUCTURES (SIMPLE NON-LINEAR MODELS)
}

\author{
Eugeniusz Bielewicz, Jarosław Górski \\ Dept of Structural Mechanics, Technical University of Gdanisk, \\ Narutowicza 11/12,80-952 Gdańsk, Poland.E-mail: jgorski@pg.gda.pl
}

Received 12 March 2001; accepted 02 Febr 2002

\begin{abstract}
Limit states of simple, spatial, non-linear models of structures with two degrees of freedom are considered. Geometric and material imperfections are taken in the form of random variables. The simulation of these random variables and the Monte Carlo technique are employed. Two possibilities in the assessment of the reliability of structures are presented: 1) Simulation of random imperfections and the Monte Carlo operation give as a result a histogram of the limit loads. Assuming that the probability distribution of the applied load is known, the structural reliability can be obtained according to the exact formula. 2) In order to obtain the histogram of the limit state of the structure, the values of the applied load are also simulated at every Monte Carlo step. The factor which amplifies the load responsible for the structure failure is derived. The set of all these factors leads to the model reliability calculation.

The estimation of the limit state of an imperfect structures can be described as a transformation of random input data into random output results. In the transformation operation the non-linear operator of the model under considerations is of the greatest significance. The effects of stable and unstable operators are discussed.
\end{abstract}

Keywords: imperfect structures, geometric and material imperfections, reliability of structures, Monte Carlo technique.

\section{Introduction}

Assessment of stability, reliability and safety of geometric and material imperfect structures belongs to the most complex problems in applied mechanics.

Stability of one-, and two-dimensional imperfect structure models has been the major concern of researchers [1-4]. These models deal mainly with elastic stability and deterministic imperfections.

The theory of probability is frequently used as a theoretical background for the analysis of random imperfections and for the reliability theory [5-7]. A similar concept forms also the basis for standard specifications [8]. In these works linear or stochastically non-linear models of structures are usually considered. It is also assumed that the limit state of the structure is known as a function of a number of random variables.

Stochastic finite elements are frequently used to describe the safety of structures and the sensitivity problems [3,9]. Non-linear models are also discussed [10].

In recent years there have appeared publications presenting an interesting concept of structure designing. The concept is based on simulation of random variables (describing loads and material properties) and on the Monte Carlo method (Marek, Guštar and Anagnos [11]). The simulation-based approach and the Monte Carlo technique are not new (see, for example, [12] and [13]), but the concept of applying these methods to practical design procedures deserves attention.
In this paper the simulation-based approach is applied to analyse non-linear models of structures. Only preliminary solutions are presented. Limit states of simple models with two degrees of freedom are considered. Geometric and material imperfections are taken in the form of random variables. The simulation of these random variables (the input data) and the Monte Carlo technique are employed. The output results lead to the assessment of the reliability of structures.

Using theses simple models, two general problems are studied.

Problem 1. The calculation of the limit state of an imperfect non-linear model of structures is, from the mathematical point of view, a transformation of random input data (imperfections) into random output results. A statistical analysis of the output results leads to determination of limit loads or limit state histograms. In the transformation procedure the non-linear operator of the model under consideration plays the most important role. The effects of stable and unstable operators are discussed.

Problem 2. Two possibilities for the reliability assessment of imperfect structures are presented. The first well-known concept takes into consideration the histogram of the structure limit loads $N_{c r}$. To obtain this histogram, simulation of random imperfections and the Monte Carlo operation are applied. Assuming that the probability distribution of the applied load $P$ is known, the structural reliability can be calculated according to the exact formula (level 3 method): 


$$
R=\operatorname{Pr}\left(N_{c r}>P\right)=\int_{-\infty}^{\infty} F_{p}(x) f_{N}(x) d x,
$$

where $F_{P}(x)$ is the cumulative distribution function of load $P$ and $f_{N}(x)$ is the probability density function of the critical load $N_{c r}$.

In the alternative approach not only the structure imperfections, but also the values of the applied load are simulated at every Monte Carlo step. Then, the factor which amplifies the simulated load responsible for the structure failure is calculated. The set of all factors obtained in this way defines the histogram of the limit state. One of the characteristics of this histogram is the reliability.

To analyse these problems, a static response of spatial non-linear models of rigid bars supported by elastic and elastic-plastic springs is considered. Stable and unstable cases are discussed. The angles of the initial bar inclinations are described using random variables. In the stable case of the bar model the angle related to the strain yield value of the spring material is taken as random. On the basis of the simulated results the histograms of the critical load and the model reliabilities are derived.

The work presents the power of the random sampling method when stochastic non-linear models are considered. The method can easily be used for analysis of realistic models of masts with elastic-plastic guys randomly loaded (for example, by wind). The results can contribute to engineering design. Some primary results of analysis of similar problems are presented in [14].

\section{Case 1: elastic solutions}

As the first example a model of a rigid bar of length $l$, hinged at the bottom and supported at the top by two linear springs of stiffness $k 1$ and $k 2$ (Fig 1) is examined. The bar is loaded by a vertical conservative force $P$. The bar position is defined by the rotation angles $\varphi$ and $\psi$. The angles of initial inclinations $\varphi_{0}$ and $\psi_{0}$ of the vertical axis are assumed.

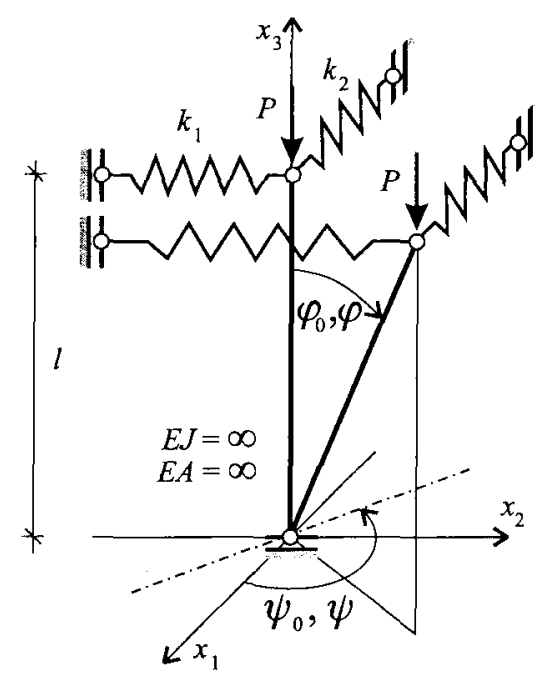

Fig 1. Model of a rigid bar supported by elastic springs (case 1)
The potential energy of the model is

$$
V(\varphi, \psi)=\frac{1}{2} k_{1} u_{5}^{2}+\frac{1}{2} k_{2} u_{2}^{2}-P u_{3}
$$

where

$$
\begin{aligned}
& u_{1}(\varphi, \psi)=l\left(\sin \varphi \sin \psi-\sin \varphi_{0} \sin \psi_{0}\right) \\
& u_{2}(\varphi, \psi)=l\left(-\sin \varphi \cos \psi+\sin \varphi_{0} \cos \psi_{0}\right) \\
& u_{3}(\varphi, \psi)=l\left(\cos \varphi_{0}-\cos \varphi\right) .
\end{aligned}
$$

From the elementary equations

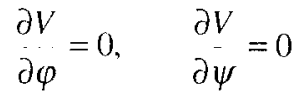

where

$$
\begin{gathered}
\partial V=k_{1} u_{1}(\cos \varphi \sin \psi)+k_{2} u_{2}(-\cos \varphi \cos \psi)-P \sin \varphi(5) \\
\partial V_{-}=k_{1} u_{1}(\cos \varphi \cos \psi)+k_{2} u_{2}(\sin \varphi \sin \psi) \\
\partial \psi
\end{gathered}
$$

the equilibrium parameters, angles $\varphi$ and $\psi$, and load related to them can be calculated.

It is easy to notice that the problem can be described as an unstable case (case 1). Thus, the known energy considerations (the second variation of potential energy is positively defined) lead to the conclusion that the stable and unstable regions are separated by the following surface

$$
\frac{\partial^{2} V \partial^{2} V}{\partial \varphi^{2} \partial \psi^{2}}-\left(\begin{array}{c}
\partial^{2} V \\
\partial \varphi \partial \psi
\end{array}\right)^{2}=0 .
$$

For example, for the fixed $\psi_{0}$ values $\left(\psi_{0}=5 \pi / 8\right)$ and the spring stiffness relations $k 2=0.1 \times k 1$ and $k 2=0.9 \times k 1$, the value of force $P$ versus angle $\varphi$ is calculated. It should be pointed out that the equilibrium paths represent complicated spatial curves. The results presented in Fig 2 are only their plain representations.

The next step in the analysis concerns the random problem.

The inclination angles $\varphi_{0}$ and $\psi_{0}$ are defined as random variables. The uniform distributions in the following intervals are assumed (Fig 1):

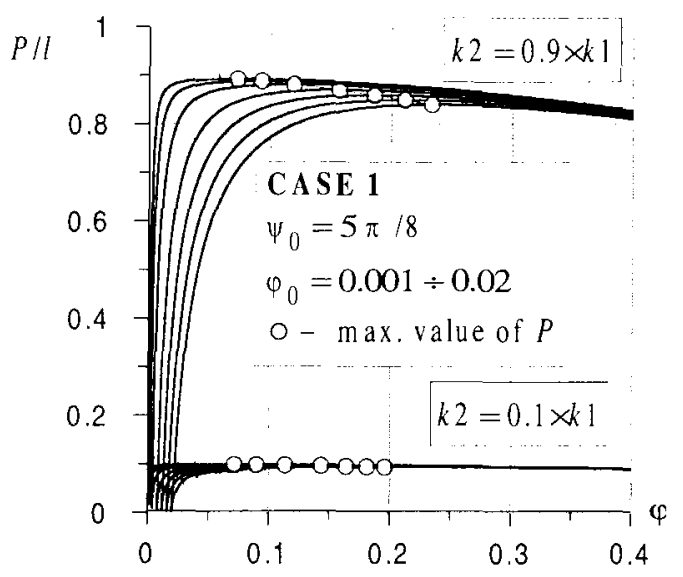

Fig 2. Case 1: equilibrium paths for the unstable model 


$$
\varphi_{0} \in(0.0,0.05\rangle, \quad \psi_{0}=\langle\pi / 2, \pi\rangle
$$

When the random inclination variables are defined, it is possible to obtain the probability distribution of the critical load $N_{c r}$. The non-linearity of the problem (lack of a one-to-one solution) creates some difficulties when analytical formulas are used. A computer calculation provides in this case real advantages.

As many as 2000 initial inclinations of the bar have been simulated ( $N R=2000$ ). For each random angle pair $\varphi_{0}$ and $\psi_{0}$ the critical value of load $N_{c r}$ is calculated. The critical load probability distribution for the identical springs stiffness $(k 2=k 1)$ is presented in Fig 3. Additionally, the expected values of the critical load $m_{N}$ and its standard deviations $D_{N}$ are calculated (Fig 3).

Assuming that the probability distribution of the applied load $P$ is stochastically independent and uniformly distributed in the interval

$$
P / l \in\{0.61,0.81\},
$$

the structure reliability $R$ can be calculated. The exact formula of 3-level method is applied (Eq 1):

$$
R=\operatorname{Pr}\left(N_{c r}>P\right)=0.9994 .
$$

Similar calculations are performed for different values of the spring stiffness: $k 2=0.95 \times k 1$. Appropriate

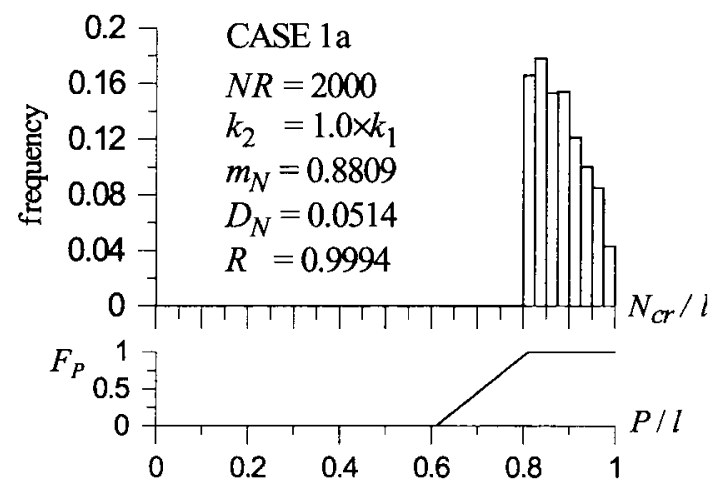

Fig 3. Case 1a: histogram of the critical load $N_{c r}$ and the probability distribution of load $P$

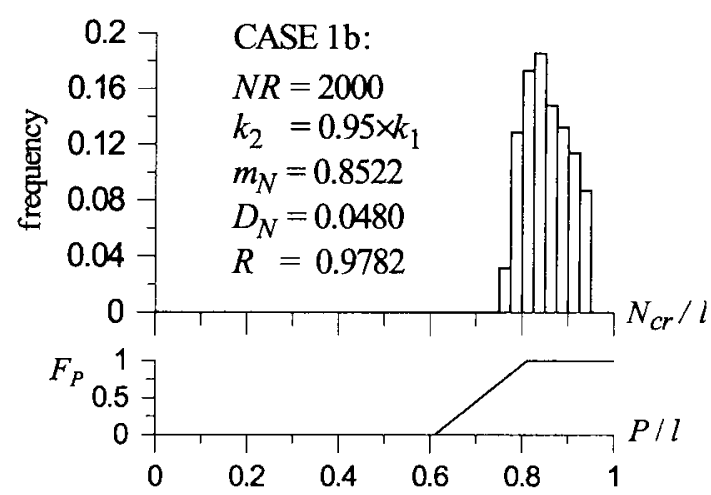

Fig 4. Case 1b: histogram of the critical load $N_{c r}$ and the probability distribution of load $P$ critical load probability distribution is presented in Fig 4.

The reliability for this case is calculated according to formula (1):

$$
R=\operatorname{Pr}\left(N_{c,}>P\right)=0.9782 .
$$

Next, an alternative concept which describes more precisely the behaviour of the model is presented.

To this end, 2000 random values of the independent, uniformly distributed random load $P$ logether with random imperfections $\varphi_{0}$ and $\psi_{0}$ are generated. At every Monte Carlo step $i(i=1,2, \ldots, N R)$ the amplification factor $\alpha_{i}$ is calculated:

$$
\alpha_{i}=N_{c r}^{i} / P_{i}
$$

A set of all factors obtained in this way defines the histogram of the limit state (Figs 5 and 6).

One of the characteristics of these histograms is the reliability. In these simple cases the following reliabilities are obtained

$$
\begin{array}{ll}
\text { case la: } & R=0.9995, \\
\text { case lb : } & R=0.9790 .
\end{array}
$$

The values are close to those calculated according to formulae (1) - see Eqs (10) and (11).

\section{Case 2: elastic-plastic solutions}

As the second example, a model of a rigid bar supported by two springs of stiffness $k 1$ and $k 2$ (Fig 7) is analysed. As in the first example the bar is loaded by a vertical conservative force $P$. The angles of initial inclinations $\varphi_{0}$ and $\psi_{0}$ of the vertical axis are assumed to be random, uniformly distributed values according to the formula (8).

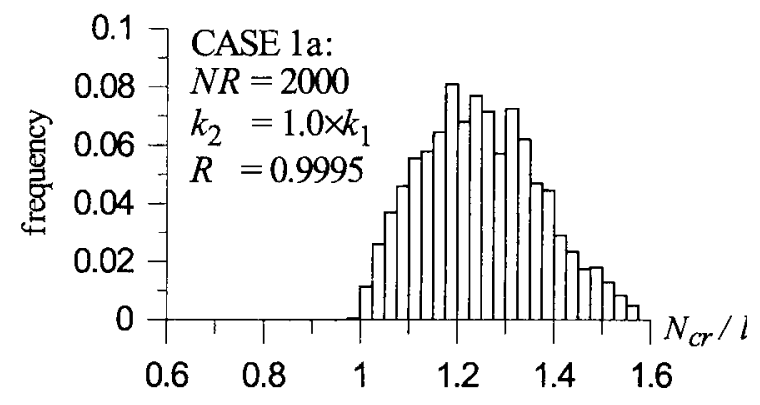

Fig 5. Case 1a: histogram of the limit state

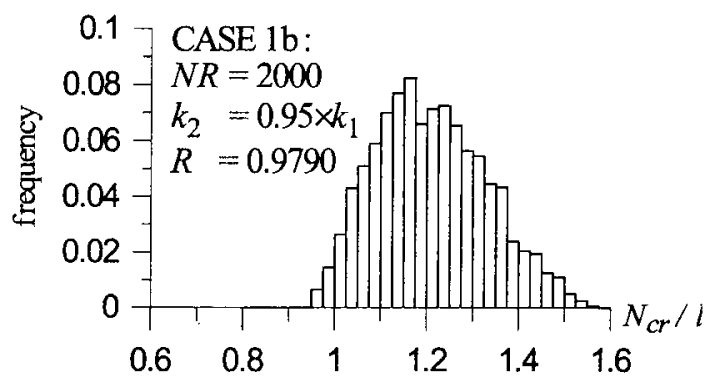

Fig 6. Case lb: histogram of the limit state 
In this case the potential energy of the model is

$$
V(\varphi, \psi)=\frac{1}{2} k_{1} \alpha_{1}^{2}+\frac{1}{2} k_{2} \alpha_{2}^{2}-P u_{3}
$$

where

$$
\begin{aligned}
& \sin \alpha_{1}(\varphi, \psi)=\sin \varphi \sin \psi-\sin \varphi_{0} \sin \psi_{0} \\
& \sin \alpha_{2}(\varphi, \psi)=-\sin \varphi \cos \psi+\sin \varphi_{0} \cos \psi_{0} \\
& u_{3}(\varphi, \psi)=l\left(\cos \varphi_{0}-\cos \varphi\right) .
\end{aligned}
$$

From the following equations

$$
\begin{aligned}
& \partial V=k_{1} \frac{\cos \varphi \sin \psi}{\sqrt{1-(\sin \varphi \sin \psi)^{2}}}\left[\sin ^{-1}(\sin \varphi \sin \psi)-\right. \\
& \left.-\sin ^{-1}\left(\sin \varphi_{0} \sin \psi_{0}\right)\right]+k_{1} \frac{\cos \varphi \cos \psi}{\sqrt{1-(\sin \varphi \sin \psi)^{2}}}
\end{aligned}
$$$$
\left[\sin ^{-1}(\sin \varphi \cos \psi)-\sin ^{-1}\left(\sin \varphi_{0} \cos \psi_{0}\right)\right]-
$$

$$
-P l \sin \varphi=0 \text {. }
$$

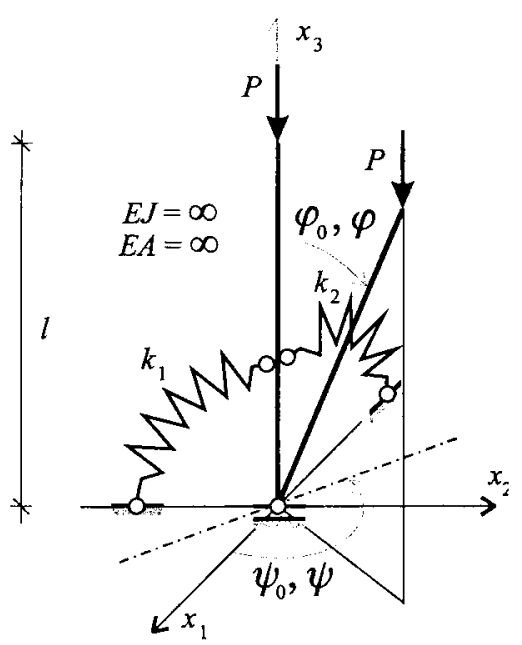

Fig 7. Model of a rigid bar supported by elastic-plastic springs (case 2)

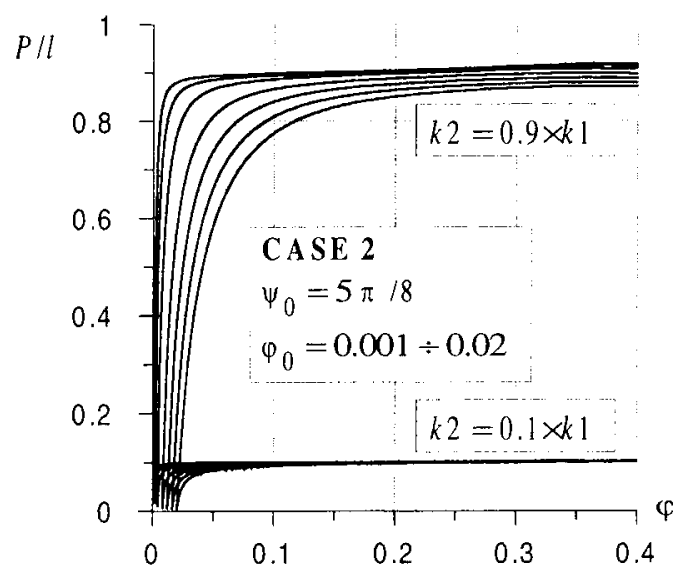

Fig 8. Case 2: equilibrium paths for the stable model

$$
\begin{aligned}
& \frac{\partial V}{\partial \psi}=k_{1} \frac{\sin \varphi \cos \psi}{\sqrt{1-(\sin \varphi \sin \psi)^{2}}}\left[\sin ^{-1}(\sin \varphi \sin \psi)-\right. \\
& \left.-\sin ^{-1}\left(\sin \varphi_{0} \sin \psi_{0}\right)\right]+k_{1} \frac{-\sin \varphi \sin \psi}{\sqrt{1-(\sin \varphi \sin \psi)^{2}}} \\
& {\left[\sin ^{-1}(\cos \varphi \cos \psi)-\sin ^{-1}\left(\sin \varphi_{0} \cos \psi_{0}\right)\right]=0}
\end{aligned}
$$

the equilibrium paths can be calculated. Some examples of these solutions are presented in Fig 8. The second model presents a stable case (case 2). It is easy to notice that the equilibrium paths in elastic range do not exhibit any maximal points.

The histograms of critical load of ideal elastic-plastic springs are considered. The following plastic bound for rotations $\varphi_{y}$ is assumed:

$$
\varphi_{y}=0.20 .
$$

On the basis of 2000 simulations, the histograms of the critical load $N_{c r}$ are derived (Figs 9 and 10).

As in case 1 , the probability distribution of load $P$ is assumed. Using the exact formula (1) the following reliabilities have been obtained:

$$
\begin{array}{ll}
\text { case } 2 \mathrm{a}: & R=0.9629, \\
\text { case } 2 \mathrm{~b}: & R=0.9340 .
\end{array}
$$

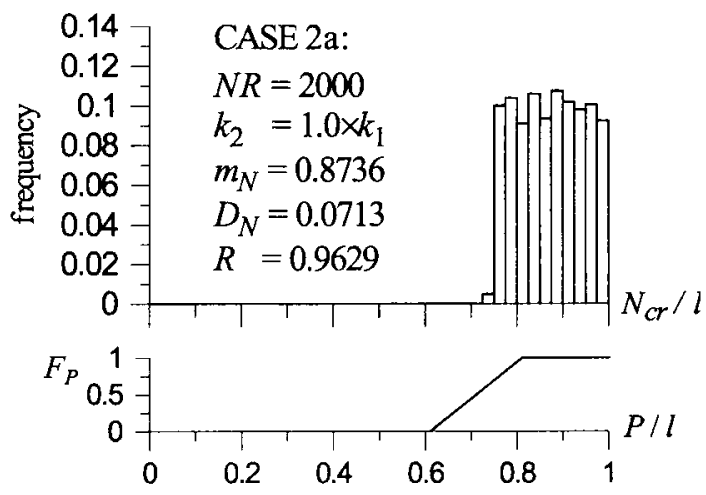

Fig 9. Case 2a: histogram of the critical load $N_{c r}$ and the probability distribution of load $P$

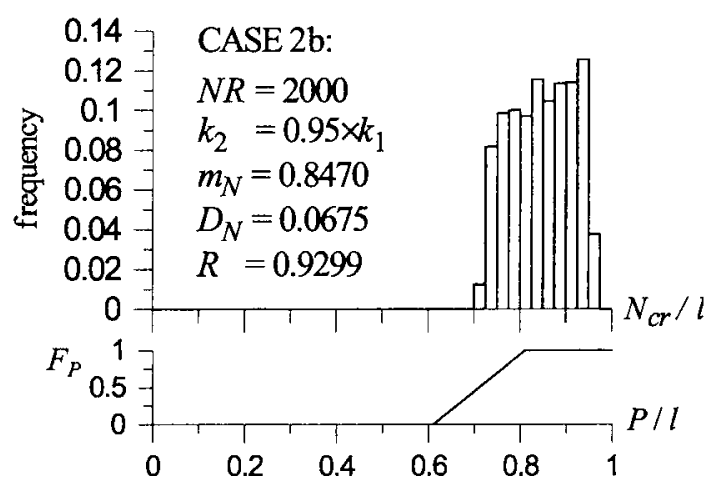

Fig 10. Case 2b: histogram of the critical load $N_{c r}$ and the probability distribution of load $P$ 


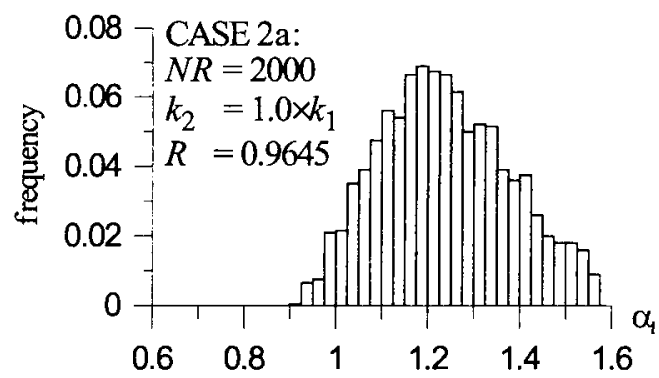

Fig 11. Histogram of the limit state (case 2a)

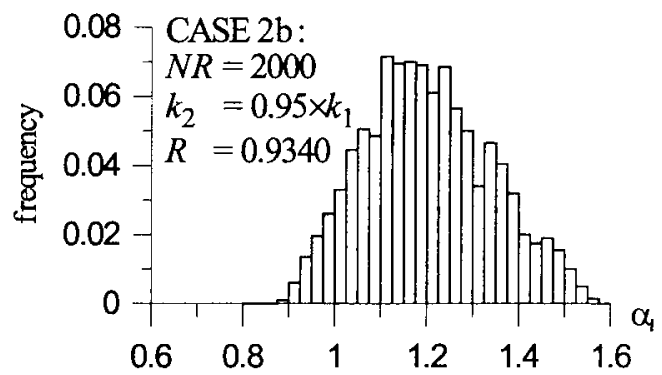

Fig 12. Histogram of the limit state (case 2b)

Alternative versions of reliabilities are also estimated. The factors $\alpha_{i}$ (Eq 12) for $N R=2000$ generated random values of load $P_{i}$ are calculated. The results are presented in Figs 11 and 12.

In these cases the reliability values compared to those obtained by the 3-level formula show significant differences:

$$
\begin{array}{ll}
\text { case } 2 \mathrm{a}: & R=0.9645, \\
\text { case } 2 \mathrm{~b}: & R=0.9340 .
\end{array}
$$

\section{Concluding remarks}

The limit states and the reliabilities of spatial nonlinear stochastic models with two degrees of freedom are considered.

Geometric and material imperfections of elastic and elastic-plastic cases are taken in the form of random variables. Analytical solutions for such problems are either unavailable or inefficient. Monte Carlo simulation appears to be the only method that can provide satisfactory solutions.

There are two ways to assess the reliability of structural models.

In the first way a histogram of critical load is calculated using Monte Carlo simulation. Then, assuming that the probability distribution of the applied load is known, the exact formula (1) is used.

In the second way the probability distribution of the applied load takes part in Monte Carlo simulation and the limit state histogram is obtained. The reliability of the structural model is one of characteristics of this histogram. The second procedure is more general and can be used when there is a combination of applied random loads.

The simulation-based methodology can be directly implemented in the analysis of real engineering structures such as masts with elastic-plastic guys. Also, twodimensional models of structures can be successfully analysed [15].

\section{Acknowledgements}

The work is partly supported by the Polish State Committee for Scientific Research through the grant No 7T07A 02116.

\section{References}

1. Gáspár Z. Imperfection-sensitivity and catastrophe theory. Collapse (Ed. by J. M. T. Thompson and G. W. Hunt), Cambridge University Press, 1983, p. 175-181.

2. Huseyin K. Nonlinear Theory of Elastic Stability. Noordhoff International Publishing, Leyden, 1975.

3. Kleiber M. and Woźniak $\mathrm{Cz}$. Nonlinear mechanics of structures. PWN, Warszawa, Kluwer Academic Publishers, Dordrecht, 1991.

4. Thompson J. M. T. and Hunt G. N. A general theory of elastic stability, John Wiley\&Sons, 1973.

5. Ditlevsen O., Madsen H. O. Structural reliability method, John Wiley \& Sons, Chichester - New York - Brisbane Toronto - Singapore, 1996.

6. Murzewski J. Reliability of engineering structures, Arkady, Warszawa, 1989 (in Polish).

7. Thoft-Christensen P. and Murotsu Y. Application of structural systems reliability theory. Springer-Verlag, Berlin, Heidelberg, New York, Tokyo, 1986.

8. IABSE Colloquium. Basis of Design and Actions on Structures, IABSE Report, Vol 74, Delft University, 1996.

9. Kleiber M., Hien T. D. The stochastic finite element method. John Wiley\&Sons, Chichester-New York-Brisbane, Toronto, Singapore, 1992.

10. Anders M., Hori M. Development of stochastic finite element method for simulation of surface earthquake fault. ASCE Journal of Applied Mechanics, 2000, Vol 3, p. 595600.

11. Marek P., Guštar M. T. Anagnos. Simulation-based reliability assessment for structural engineers. CRC Press, Boca Raton, New York - London - Tokyo, 1996.

12. Augusti G., Baratta A., Casciati F. Probabilistic methods in structural engineering. London, New York, Chapman and Hall, 1984.

13. Hurtado J. E., Barbat A. H. Monte Carlo techniques in computational stochastic mechanics. Archives of Computational Method in Engineering, Vol 5. 1, 1998, p. 3-30.

14. Walukiewicz H., Bielewicz E., Górski J. Discrete spatial simulations in structural mechanics and in environmental problems. Advances in Engineering Software, 1998, Vol 29, No 7-9, p. 723-731.

15. Górski J. Simulation-based nonlinear analysis of imperfect structures. Archives of Civil Engineering, No 47, Vol 1, 2001, p. 3-18. 